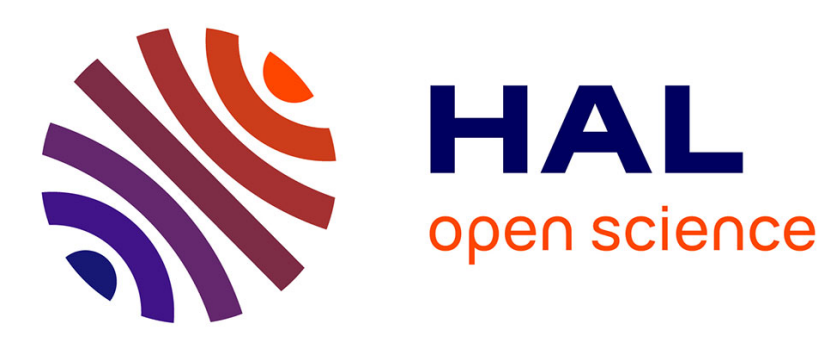

\title{
First case of brucellosis caused by an amphibian-type Brucella
}

\author{
Nicolas Rouzic, Ludovic Desmier, Marie-Estelle Cariou, Eugénie Gay, Jeffrey \\ T Foster, Charles H D Williamson, François Schmitt, Mikael Le Henaff, Alain \\ Le Coz, Aurélien Lorléac'h, et al.
}

\section{To cite this version:}

Nicolas Rouzic, Ludovic Desmier, Marie-Estelle Cariou, Eugénie Gay, Jeffrey T Foster, et al.. First case of brucellosis caused by an amphibian-type Brucella. Clinical Infectious Diseases, In press, pp.ciaa1082. 10.1093/cid/ciaa1082 . hal-02951189

\section{HAL Id: hal-02951189 \\ https://hal.umontpellier.fr/hal-02951189}

Submitted on 28 Sep 2020

HAL is a multi-disciplinary open access archive for the deposit and dissemination of scientific research documents, whether they are published or not. The documents may come from teaching and research institutions in France or abroad, or from public or private research centers.
L'archive ouverte pluridisciplinaire HAL, est destinée au dépôt et à la diffusion de documents scientifiques de niveau recherche, publiés ou non, émanant des établissements d'enseignement et de recherche français ou étrangers, des laboratoires publics ou privés. 


\title{
First case of brucellosis caused by an amphibian-type Brucel/a
}

\author{
Running title : $1^{\text {st }}$ infection by frog Brucella in human
}

Nicolas Rouzic ${ }^{1}$ (MD), Ludovic Desmier²,3 ${ }^{2,3 a r i e-E s t e l l e ~ C a r i o u ~}{ }^{4}$ (PharmD), Eugénie Gay ${ }^{4}$ (MD), Jeffrey T. Foster ${ }^{5}(\mathrm{PhD})$, Charles H.D. Williamson ${ }^{5}(\mathrm{PhD})$, François Schmitt ${ }^{4}$ (PharmD, PhD), Mikael Le Henaff ${ }^{6}$ (MD), Alain Le $\mathrm{Coz}^{6}(\mathrm{MD})$, Aurélien Lorléac'h ${ }^{1}(\mathrm{MD})$, Jean-Philippe Lavigne ${ }^{2,3}(\mathrm{MD}, \mathrm{PhD})$, David O'Callaghan ${ }^{2,3}(\mathrm{PhD})$ and Anne Keriel ${ }^{2,3 *}(\mathrm{PhD})$

${ }^{1}$ Unité de Médecine Interne - Maladies Infectieuses, Groupe Hospitalier Bretagne Sud, Lorient, France

${ }^{2}$ Centre National de Référence (CNR) des Brucella, CHU de Nîmes, Nîmes, France

${ }^{3}$ VBMI, U1047, INSERM, Université de Montpellier, Nîmes, France

${ }^{4}$ Laboratoire de biologie médicale, Groupe Hospitalier Bretagne Sud, Lorient, France

${ }^{5}$ The Pathogen \& Microbiome Institute (PMI), Northern Arizona University, Arizona, USA

${ }^{6}$ Service de Pneumologie, Groupe Hospitalier Bretagne Sud, Lorient, France

Keywords : brucellosis, Brucel/a, amphibian, atypical, pulmonary infection

Corresponding author: Anne Keriel, anne.keriel@,inserm.fr, Phone: +33466028157

Alternate corresponding author: David O’Callaghan, david.o-callaghan@umontpellier.fr, Phone: +33466028146 
ABSTRACT: We report the first case of brucellosis caused by an isolate whose genome is identical that of a frog isolate from Texas, demonstrating the zoonotic potential of amphibian-type Brucel/a. Importantly, with such atypical Brucel/a, correct diagnosis cannot be performed using routine serological tests or identification methods. 
Brucellosis, an infectious disease caused by members of the Brucella genus, remains one of the most common bacterial zoonotic diseases worldwide and is endemic in most developing countries, mainly in areas with extensive farming [1]. Brucella are Gram-negative facultative intracellular bacteria that can infect many organs and soft tissues. Brucel/a melitensis (goats) is the predominant species causing human brucellosis, but $B$. abortus (cattle) or B. suis (swine) may also be involved. Transmission is commonly due to the consumption of unpasteurized dairy products or direct exposure to diseased animals. Metropolitan France is officially free from bovine brucellosis since 2005 and autochthonous human infections are extremely rare. Human brucellosis is a chronic debilitating infection with non-specific clinical presentation, often presenting as a flu-like syndrome. Symptoms occur after an incubation period of 2 weeks, or up to several months in some cases.

Mid-January 2019 (day 0), a French 28-years-old patient was hospitalized in the general hospital of Lorient (France) for an exploration of polyadenopathies associated with multiple pulmonary condensations.

Four months earlier, the patient had reported a left cervical lymphadenopathy, associated with general degradation in condition and weight loss (8 $\mathrm{kg}$ in 3 months). A cervical ultrasound performed on day -30 confirmed fluid accumulation in the left cervical region and revealed two others in the maxillary region. The patient also reported intense night sweats (without known fever) and a loose cough that resolved spontaneously. The patient, also affected by Wilson's disease, previously suffered from hypertension requiring multiple ligations of varicose veins. His treatment at day 0 was: propranolol, pyridoxine, Dpenicillamine, paroxetine and hydroxyzine.

On admission (day 0), the patient had altered general status, moderate dyspnea without cough and night fever $\left(39^{\circ} \mathrm{C}\right)$. Clinical examination confirmed a left cervical lymphadenopathy. Heart and lungs auscultations were normal. Initial laboratory tests (day 1) showed a C-reactive protein level of $37 \mathrm{mg} / \mathrm{L}$, indicative of an active infection, but total leukocyte count of within the normal range $(5 \times 109 / \mathrm{L}$ with $60 \%$ polymorphonuclear cells. 
HIV serology was negative. Blood cultures were negative after five days incubation. A cervico-thoracic and abdominal CT scan revealed multiple mediastinal adenopathies, pulmonary parenchymal condensations, cystic emphysematous lesions in the right upper lobe and splenomegaly (Figure S1).

On day 6, a left cervical lymph node was surgically removed, which triggered fever in the patient. Histopathologic examination of the excised tissue revealed an aspecific epitheliogigantocellulary granuloma with caseous necrosis, without acid-fast bacilli (later confirmed by negative mycobacterial culture). Aerobic cultures of the tissue were positive after $48 \mathrm{~h}$ incubation on Chocolate + polyvitex, Drigalski and Columbia $+5 \%$ sheep blood agar plates (bioMérieux). Colonies were about $1 \mathrm{~mm}$ in diameter, circular, glistening, smooth, nonpigmented and non-haemolytic. Culture of endobronchial aspiration from the patient in a Mycobacteria Growth Indicator Tube (Becton Dickinson) amplified bacteria that, upon subculturing on a Columbia plate, gave colonies with similar appearance. Bacteria were Gramnegative coccobacilli and showed positive reactions for catalase and cytochrome-oxidase, but negative result for indole production. The bacterial isolate was analyzed by MALDI-TOF MS using a Biotyper (Bruker Daltonics) with the version 7 (V7) of the IVD (In Vitro Diagnostics) database. The identification result was Ochrobactrum anthropi (score 1.49). Because of the low score and inconsistency with phenotypic observations, the isolate was re-analyzed using Bruker's Security-Relevant Library database and the identification result was Brucel/a melitensis (score 2.23). A treatment with oral doxycycline (200 $\mathrm{mg}$ once daily) and rifampicin $(15 \mathrm{mg} / \mathrm{Kg} /$ day $)$ was thus initiated and the isolate sent to the national reference centre (CNR Brucel/a) for confirmation of identification and further analysis. MALDI-TOF MS re-analysis using a Vitek MS (bioMérieux) with the IVD database V3.2 confirmed that the isolate belongs to the Brucella genus. However, the use of a recently developed, more complete, RUO (Research Use Only) database [2] rather suggested that it was a B. inopinata (score 99.9\%). A blood sample drawn on day 1 was also submitted to several Brucel/a serological tests: Rose Bengal (Brucelloslide-Test, bioMérieux), Wright agglutination 
(Brucella Wright, Bio-Rad), ELISA (Virclia IgM or IgM, Vircell) and immunocapture test (BrucellaCapt, Vircell). All tests were negative.

After 6 weeks of antibiotic therapy, the patient reported resolution of his malaise and fatigue but presented a left cervical abscess under a surgical scar. Culture of an aspirate from this abscess was negative, but Brucella DNA was detected by PCR amplification of the Brucel/a-specific sequence IS711 [3]. The persistence of deep-seated lymphadenopathy associated with multiple pulmonary condensations was confirmed by a CT scan showing an unsatisfactory progression. The antibiotic treatment was thus prolonged for a total of 3 months, to which the patient responded favorably, remaining clear of symptoms 6 months after the end of treatment.

An epidemiological survey was conducted to try identifying the origin of the infection. The patient declared no travel abroad in the past five years and no consumption of unpasteurized dairy products. However, as part of his professional activity (animal keeper specializing in exotic animals), he reported many direct contacts with reptiles, amphibians, rodents or birds, several imported from abroad.

The bacterial isolate was characterized using a Bruce-Ladder multiplex PCR [4] on genomic DNA and phylogenic positioning based on MALDI-TOF MS spectra. This revealed that this isolate is most similar to the $B$. inopinata-like isolates $\mathrm{BO} 2$ and $\mathrm{B} 13-0095$, isolated from a patient with severe pulmonary infection [5] and from a frog [6], respectively (Figures S2 and S3). The isolate, thereafter named BO3 (for Brucella inopinata-like 3), showed susceptibility to all tested antibiotics, as for B. melitensis and the other B. inopinata(-like) strains (Table S1). Several features however clearly distinguished BO3 from classical Brucel/a spp. First, slide agglutination using a polyclonal serum anti-Brucella (ANSES, Maisons-Alfort, France) was negative. We also found that BO3 is highly motile, like other $B$. inopinata(-like) strains [6], and flagellated (Figure S4), which is completely novel for this bacterial genus.

Sequencing of BO3 genome revealed two chromosomes of 2,181,202 bp and 1,197,108 
bp, respectively, with a GC content of $57.11 \%$, similar to other Brucella species. No plasmid was detected. A genome-based phylogenetic tree confirmed that $\mathrm{BO} 3$ belongs to the group of 'atypical' Brucella, together with the human isolates BO1 (USA, 2005 [7]) and BO2 (Australia, 2007 [5]) and a growing number of frog isolates [8], and is extremely closely related to the frog isolate B13-0095 [6] (Figure 1). Annotation of the BO3 genome using PATRIC https://www.patricbrc.org identified the virB operon (encoding a type IV secretion system) on chromosome 2. As with strains BO2 and B13-0095, the wbk operon (encoding the perosamine-based O-antigen in classical Brucella species) is absent from $\mathrm{BO} 3$ genome and, instead, a gene cluster encoding a system for L-rhamnose uptake and catabolism is present, suggesting an atypical lipopolysaccharide. BO3 DNA also contains a cluster of genes involved in ectoine catabolism (Figure S5), a genetic feature specific to amphibian-type Bruce/la $[6,8]$. When calculating genome-to-genome distance, the DNA-DNA hybridization (DDH) estimate between strains BO3 and B13-0095 was 99.9\%, indicating that these genomes are identical. As a comparison, the $\mathrm{DDH}$ estimate between $\mathrm{BO} 3$ and $\mathrm{BO} 2$ was $89.8 \%, 84.9 \%$ with BO1, 83.3\% with 09RB8471 (African bullfrog [9]), 83.2\% with 141012304 (bluespotted ribbontail ray [10]), 81.5\% with 10RB9215 (African bullfrog) and 81.4\% with 09RB8910 (African bullfrog). Altogether, our analyses indicate that this new strain $\mathrm{BO} 3$ is identical to the $B$. inopinata-like strain B13-0095 that was isolated from a PacMan frog in Texas in 2010 [6].

This is thus the first report of human brucellosis caused by an amphibian-type Brucel/a. The scarcity of reports may be due to the difficulty to identify them as Brucel/a spp. Because of their rapid growth rate on plates $(<24 \mathrm{~h})$ and because phenotypic identification tests (such as API 20NE) misidentify them as Ochrobactrum spp. with a high confidence level [6,11]. Moreover, since some strains have an LPS that is chemically and antigenically different from that of classical Brucella, infection cannot be detected using commonly available serological tests and the isolates cannot be identified using specific typing serum. Finally, when using MALDI-TOF MS as an identification method, it should be noted that the Biotyper (Bruker), 
with its Security Relevant database, and the Vitek MS (bioMérieux), with its most recent IVD database ( $\geq$ V3.2), would identify such atypical isolates as B. melitensis or Brucella spp., respectively. Correct identification as $B$. inopinata(-like) is currently only possible using the latest version ( $\geq$ V4.15) of the RUO database Saramis on a Vitek MS [2].

The animal reservoir(s) of $B$. inopinata(-like) strains remain to be identified. Isolation from different animals (either wild caught or kept in zoological collections, pet store or by private breeder [8]) on several continents suggests that these bacteria are widespread. Recent isolation from a ray also suggests that all vertebrates (including fish and reptiles) may be suitable hosts. We could not detect traces of Brucella in cultures of samples taken from the patient's pets (mainly snakes), suggesting that exposure occurred during his past professional activity as an exotic animal keeper (notably, the patient declared being in contact with PacMan frogs). We speculate that $B$. inopinata(-like) bacteria may be part of the normal flora of some animals and become opportunistic pathogens for their host upon unfavorable conditions such as stress caused by transport (importation of wild-caught animals), changes in breeding facilities (i.e. quarantine protocols) or contact with other species creating opportunities for cross-species infection. Also, pathogenicity for humans may be linked to lowered immunocompetency.

Medical practitioners and diagnostic laboratories should be aware of the existence of these pathogens. Prevention measures should be taken, especially for exotic animal keepers and veterinarians, who should limit direct contact with potentially infected animals. 


\section{ACKNOWLEDGMENTS}

This work was supported by the Institut National de la Santé et de la Recherche Médicale (Inserm), Université de Montpellier and Santé Publique France. We are grateful to Chantal Cazevieille (Electron Microscopy Platform, Montpellier RIO Imaging) for the visualisation of the BO3 cells. We are also very grateful to MVDr Paredes (Clinique Vétérinaire Copernic, Vannes, France) for sampling the patient' reptiles and to Fanny Broussard for the analysis of the animals samples.

\section{COMPLIANCE WITH ETHICAL STANDARDS}

Conflict of interest: All authors do not declare any conflict of interest.

Ethical approval: Publication of case reports does not require approval by an Institutional Review Board.

Informed consent: The patient gave his written informed consent for the publication of this case report.

Nucleotide sequence accession numbers and deposition

The genome sequences of isolate $\mathrm{BO} 3$ are available from the GenBank database under accession numbers CP047232-CP047233 (BioProject PRJNA597040). 


\section{$\underline{\text { REFERENCES }}$}

1. Godfroid J, Al Dahouk S, Pappas G, et al. A “One Health” surveillance and control of brucellosis in developing countries: Moving away from improvisation. Comp Immunol Microbiol Infect Dis 2013;

2. Mesureur J, Arend S, Cellière B, et al. A MALDI-TOF MS database with broad genus coverage for species-level identification of Brucella. PLoS Negl Trop Dis 2018;

3. Sanjuan-Jimenez R, Morata P, Bermúdez P, Bravo MJ, Colmenero JD. Comparative Clinical Study of Different Multiplex Real Time PCR Strategies for the Simultaneous Differential Diagnosis between Extrapulmonary Tuberculosis and Focal Complications of Brucellosis. PLoS Negl Trop Dis 2013;

4. López-Goñi I, García-Yoldi D, Marín CM, et al. Evaluation of a multiplex PCR assay (Bruce-ladder) for molecular typing of all Brucel/a species, including the vaccine strains. J Clin Microbiol 2008;

5. Tiller R V, Gee JE, Lonsway DR, et al. Identification of an unusual Brucella strain (BO2) from a lung biopsy in a 52 year-old patient with chronic destructive pneumonia. BMC Microbiol 2010; 10:23.

6. Soler-Lloréns PF, Quance CR, Lawhon SD, et al. A Brucel/a spp. Isolate from a PacMan Frog (Ceratophrys ornata) Reveals Characteristics Departing from Classical Brucellae. Front Cell Infect Microbiol 2016; 6:116.

7. De BK, Stauffer L, Koylass MS, et al. Novel Brucel/a Strain (BO1) Associated with a Prosthetic Breast Implant Infection. J Clin Microbiol 2008; 46:43-49.

8. Muhldorfer K, Wibbelt G, Szentiks CA, et al. The role of "atypical" Brucella in amphibians: are we facing novel emerging pathogens? J Appl Microbiol 2016;

9. Al Dahouk S, Köhler S, Occhialini A, et al. Brucel/a spp. of amphibians comprise genomically diverse motile strains competent for replication in macrophages and survival in mammalian hosts. Sci Rep 2017; 7:44420.

10. Eisenberg T, Ri??e K, Schauerte N, Geiger C, Blom J, Scholz HC. Isolation of a novel 
atypical Brucel/a strain from a bluespotted ribbontail ray (Taeniura lymma). Antonie van Leeuwenhoek, Int J Gen Mol Microbiol 2016; :1-14.

11. Tiller R V, Gee JE, Frace MA, et al. Characterization of novel Brucella strains originating from wild native rodent species in North Queensland, Australia. Appl Environ Microbiol 2010; 76:5837-5845.

12. Scholz HC, Revilla-Fernández S, Dahouk S Al, et al. Brucel/a vulpis sp. Nov., isolated from mandibular lymph nodes of red foxes (vulpes vulpes). Int J Syst Evol Microbiol 2016; 
Figure 1: The amphibian-type isolate B03 belongs to the emerging group of atypical Brucella. BO3 genomic DNA was extracted (DNeasy UltraClean Microbial Kit, Qiagen) and sequenced using MinION (Oxford Nanopore Technologies) and Illumina MiSeq (Illumina) platforms. Sequence reads were de novo assembled with Unicycler v0.4.7, processed with Circlator v1.5.5 and polished with Pilon v1.23) resulting in a high quality genome. A genome-based tree was then constructed using the reference strains of all Brucel/a species and selected 'atypical' strains. Assemblies were aligned to a reference genome with NUCmer; core genome single nucleotide polymorphisms were called within NASP; and a maximum likelihood phylogeny was inferred with IQ-TREE. This phylogenetic tree shows clustering of BO3 within the group of "atypical" Brucel/a, together with B. inopinata BO1 [7], B. inopinata(-like) BO2 [5], and isolates from Australian rodents (NF2653 and 83/13 [11]), red foxes (F60 and F965 [12]), a bluespotted ribbontail ray [10] and frogs (B13-0095, 09RB8910, 10RB9215 and 09RB8471 [6,9]). 


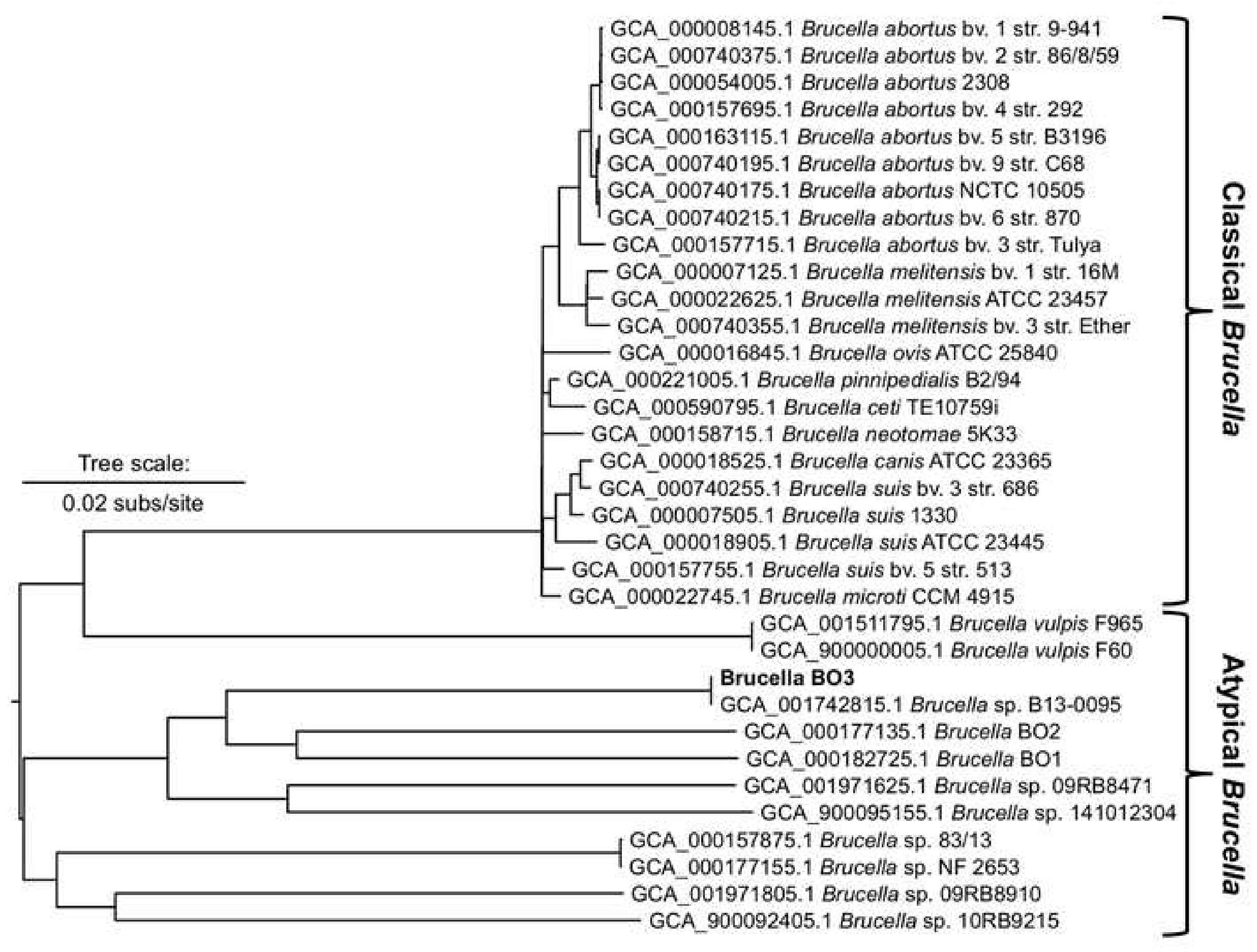




\section{Rouzic N. et al., manuscript for Clinical Infectious Diseases}

\section{SUPPLEMENTARY MATERIAL}

Figure S1: Initial CT scan showing hilar and subcarinal adenopathies (A) and parenchymal condensations (B).
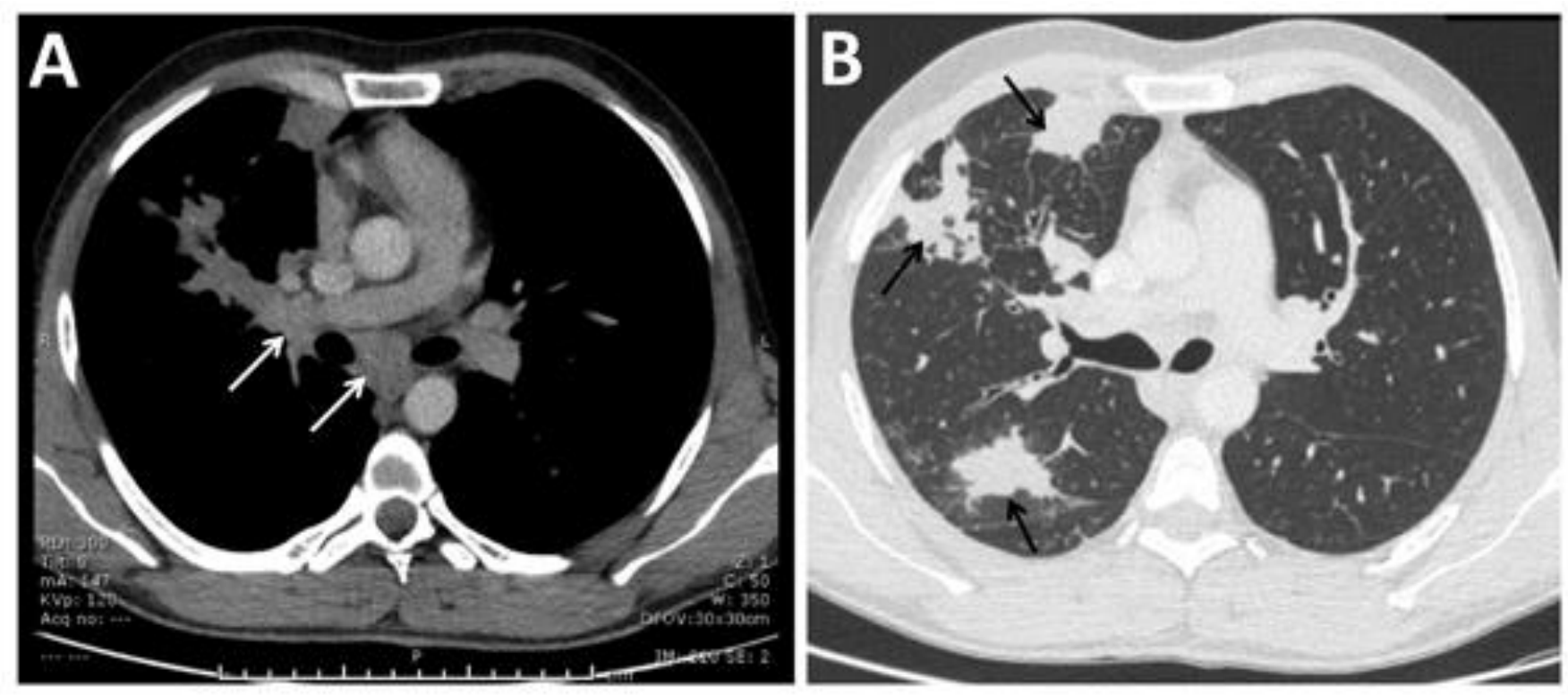
Figure S2: Identification of the patient bacterial isolates as Brucella. inopinata-like. Bruce-Ladder multiplex PCR was performed on two bacterial isolates BRSO-2019-086 and BRSO-2019-089, recovered after puncture of two different lymph nodes in the patient at day 6 and -7, respectively. Bruce-Ladder profiles were compared to classical (B. melitensis, B. abortus, B. suis and B. canis) or atypical (B. inopinata strain BO1 or B. inopinata-like strains BO2 and frog B13-0095) Brucel/a isolates after agarose gel electrophoresis. Left $=$ molecular-weight size marker $($ size in $b p) . \varnothing=$ negative control.

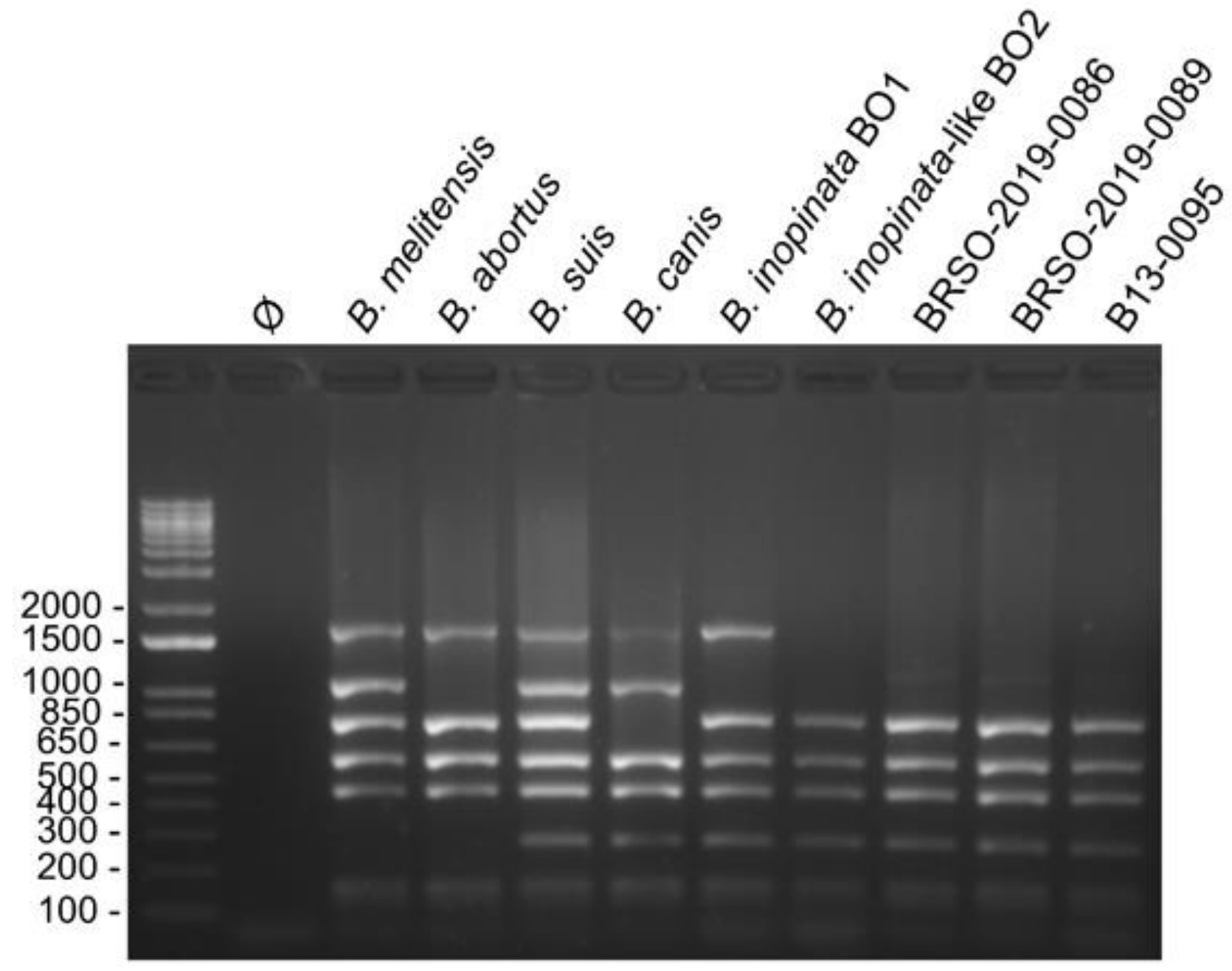


Figure S3: Proximity of MALDI-TOF MS spectra. Dendrogram constructed from MALDI-TOF MS spectra using SARAMIS (bioMérieux). Similarity between spectra is quantified by the proportion of common masses; the dotted line (65\%) indicating a threshold above which bacteria are considered as significantly different. This representation shows the distance between Brucella strains belonging to the classical or to the atypical clade of this genus. This highlights clustering of the two patient isolates BRSO-2019-086 and BRSO-2019-089 (arrows) with the group of "atypical" Brucella, and confirms the clonality of these two isolates, which were thereafter considered as a single strain named BO3 (for Brucella inopinata-like 3).

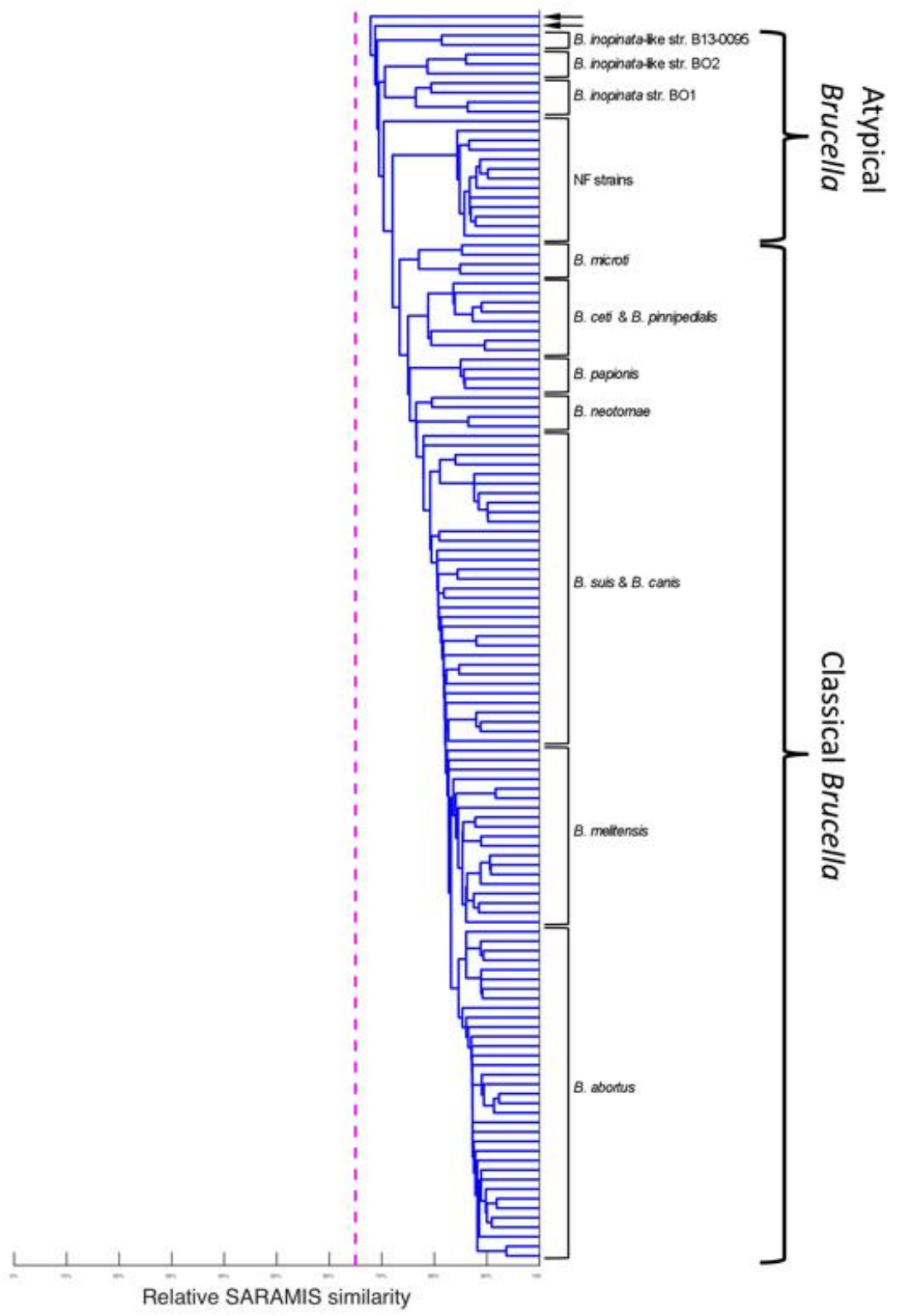


Table S1: Antimicrobial susceptibility tests by disk diffusion assay. Bacterial isolates were plated on Mueller-Hinton agar with 5\% sheep blood (bioMérieux), as established by the Clinical Laboratory Standards Institute and considering suggestions for Gram-negative bacteria. The cut-offs (in brackets) and the measured inhibition diameters are indicated in $\mathrm{mm} .16 \mathrm{M}=B$. melitensis biovar 1 reference strain, $\mathrm{BO} 1=B$. inopinata clinical isolate [1], $\mathrm{BO} 2=$ B. inopinata-like clinical isolate [2], $\mathrm{BO}=$ B. inopinata-like clinical isolate (this case), B13-0095 = B. inopinata-like frog isolate [3] .

\begin{tabular}{|c|c|c|c|c|c|c|c|c|c|}
\hline & $\begin{array}{l}\text { Rifampicin } \\
\qquad(\geq 18)\end{array}$ & $\begin{array}{l}\text { Tetracycline } \\
\qquad(\geq 15)\end{array}$ & $\begin{array}{c}\text { Gentamycin } \\
\quad(\geq 17)\end{array}$ & $\begin{array}{c}\text { Amikacin } \\
(\geq 16)\end{array}$ & $\begin{array}{l}\text { Trimethoprim }+ \\
\text { Sulfamethoxazol } \\
\qquad \geq 14)\end{array}$ & $\begin{array}{l}\text { Ciprofloxacin } \\
\qquad(\geq 24)\end{array}$ & $\begin{array}{l}\text { Ofloxacin } \\
(\geq 22)\end{array}$ & $\begin{array}{l}\text { Amoxicillin } \\
\quad(\geq 19)\end{array}$ & $\begin{array}{c}\text { Amoxicillin }+ \\
\text { Clavulanic } \\
\text { acid } \\
(\geq 19)\end{array}$ \\
\hline $16 \mathrm{M}$ & 40 & 45 & 32 & 32 & 42 & 40 & 32 & 52 & 49 \\
\hline B01 & 32 & 43 & 23 & 22 & 28 & 35 & 32 & 40 & 40 \\
\hline B02 & 32 & 50 & 24 & 24 & $20 / 25$ & $24 / 24$ & $24 / 23$ & 50 & 42 \\
\hline B03 & 30 & 40 & 22 & 22 & $16 / 25$ & $30 / 30$ & $30 / 26$ & 40 & 32 \\
\hline $\begin{array}{l}\text { B13- } \\
0095\end{array}$ & 32 & 40 & 22 & 22 & $15 / 18$ & $30 / 29$ & $24 / 23$ & 40 & 36 \\
\hline
\end{tabular}


Figure S4: Brucella B03 isolate is flagellated and motile. A) Swimming assays on soft-agar plates showing that $\mathrm{BO} 3$ is motile. As a control, strains $\mathrm{BO} 1$ and $\mathrm{BO} 2$ are also motile while $B$. melitensis strain 16M (classical Bruce/la) is not, in agreement with previous observations [3]. B) Visualization of BO3 by electron microscopy revealing bacterial cells with a mean average diameter of $0.5 \mu \mathrm{m}$ and a length of 1 $\mu \mathrm{m}$, some of them with a polar flagellum $($ scale bar $=500 \mathrm{~nm})$.

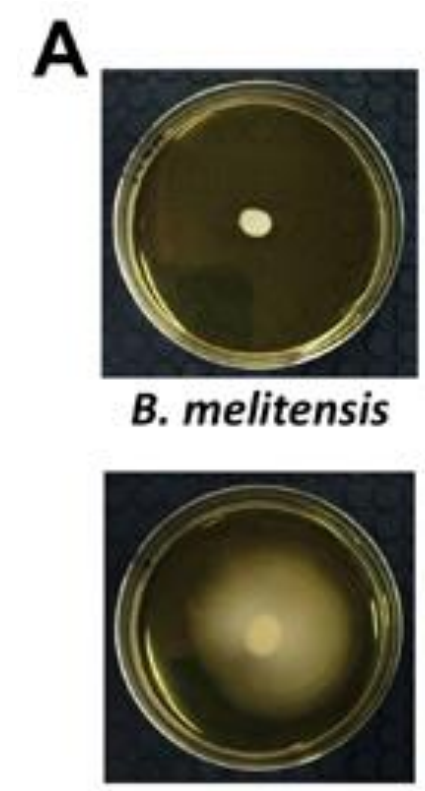

BO2

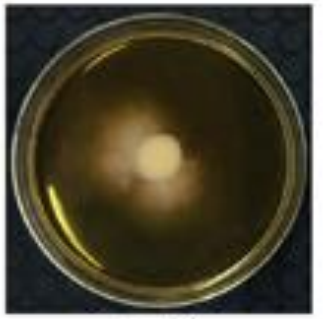

B01

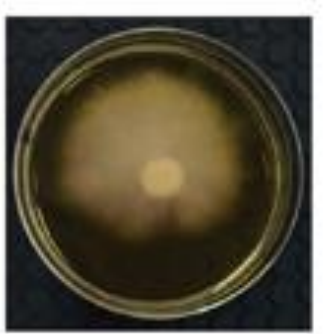

BO3
B

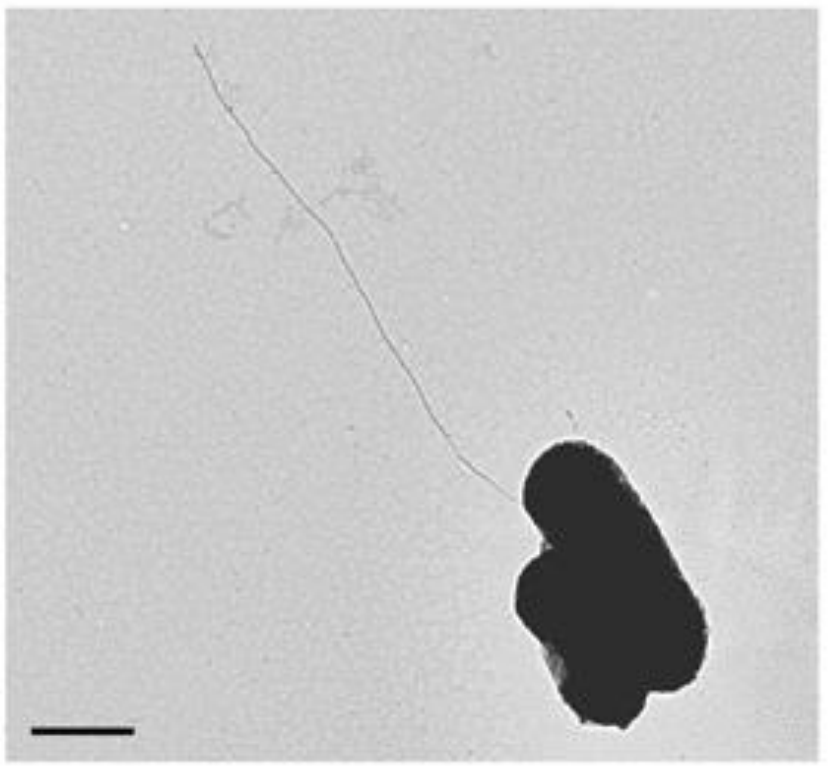


Figure S5: Detection of the Ectoin genes cluster by PCR. A) Specific PCR strategy: two independent reactions were performed using the primers pairs $\mathrm{F}$ (5'-actggctcttcctcaagac-3') and R1 (5'accgacaacatcgcaatc-3') or F and R2 (5'-gcaagttcttcgacccatc-3') to amplify either a 370 bp fragment common to all Brucel/a species (as a positive control) or a $1234 \mathrm{bp}$ fragment when the bacterial genomic DNA contains the Ectoin genes cluster, respectively. B) PCR results for the two patient's isolates (BRSO2019-086 and BRSO-2019-089) compared to other Brucella isolates (B. melitensis 16M, B. inopinata strain BO1, B. inopinata-like strains BO2 and frog B13-0095) after agarose gel electrophoresis. Left = molecular-weight size marker (size in bp). $\varnothing=$ negative controls.

A

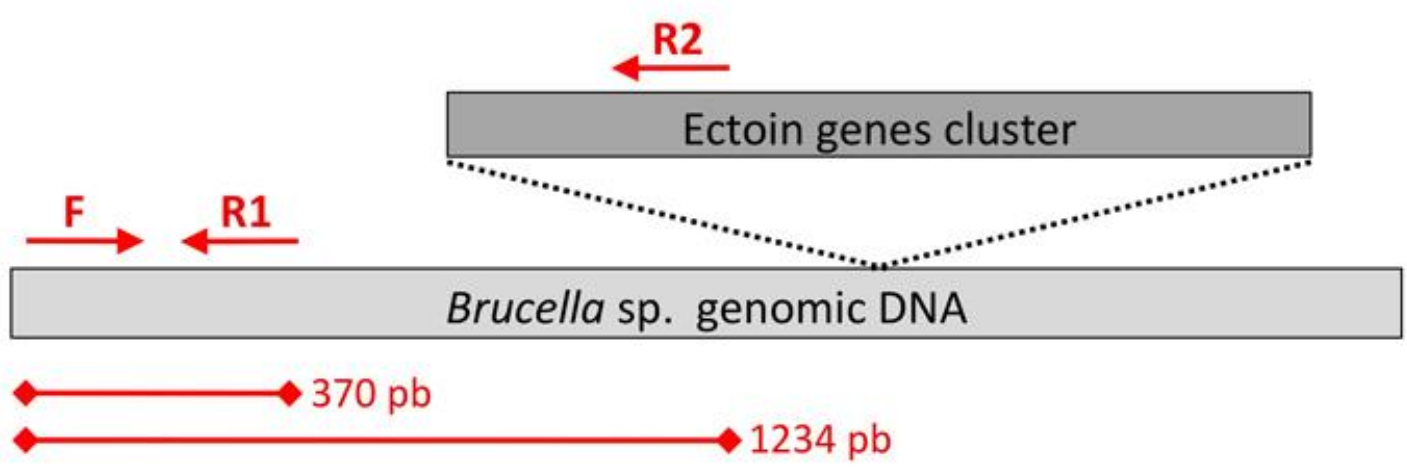

B

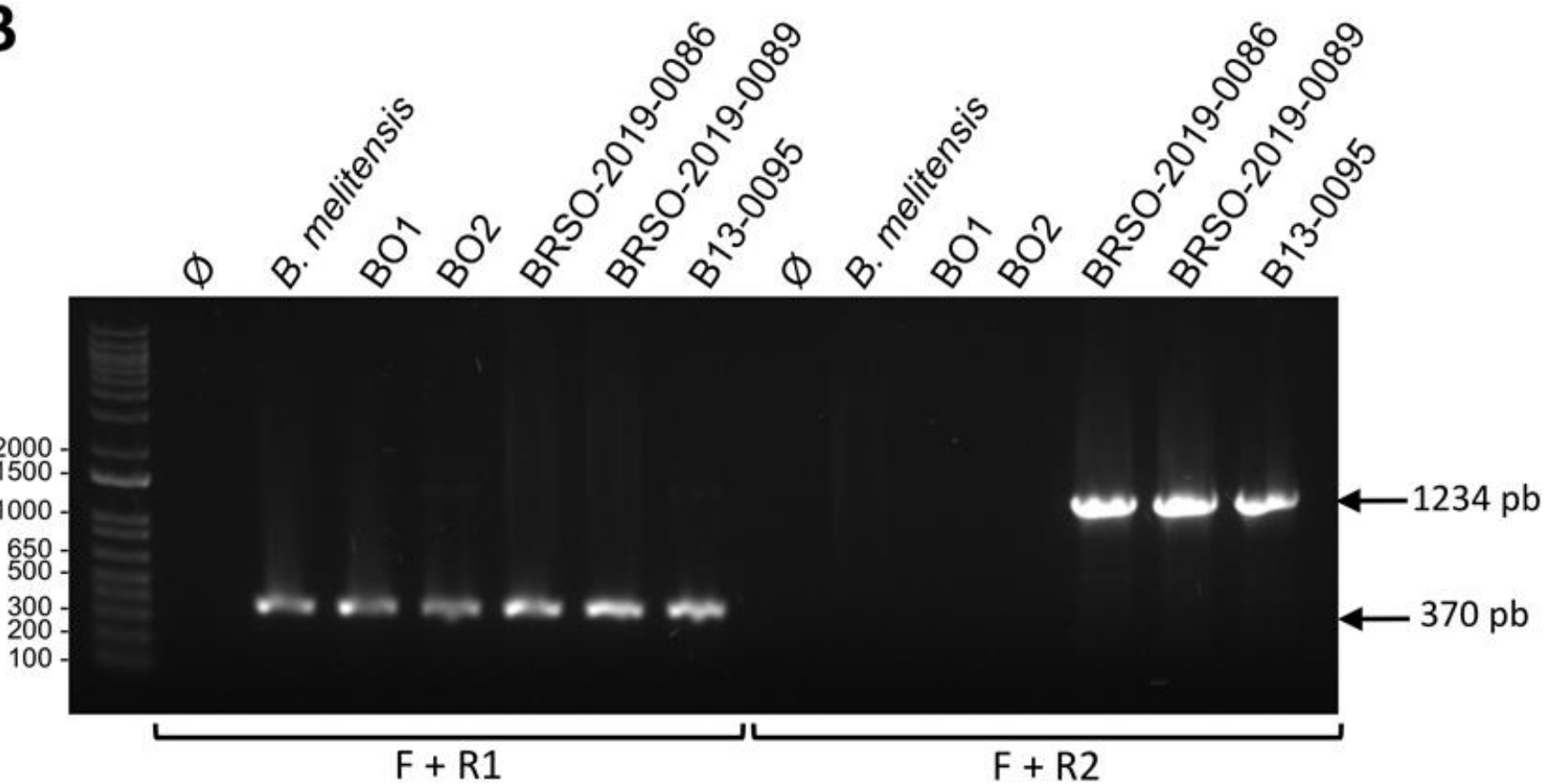




\section{$\underline{\text { REFERENCES }}$}

1. De BK, Stauffer L, Koylass MS, et al. Novel Brucel/a Strain (BO1) Associated with a Prosthetic Breast Implant Infection. J Clin Microbiol 2008; 46:43-49.

2. Tiller R V, Gee JE, Lonsway DR, et al. Identification of an unusual Brucella strain (BO2) from a lung biopsy in a 52 year-old patient with chronic destructive pneumonia. BMC Microbiol 2010; $10: 23$.

3. Soler-Lloréns PF, Quance CR, Lawhon SD, et al. A Brucella spp. isolate from a Pac-Man frog (Ceratophrys ornata) reveals characteristics departing from classical brucellae. Front Cell Infect Microbiol 2016; 